\title{
Control of gene expression by CRISPR-Cas systems
}

\author{
David Bikard* and Luciano A. Marraffini*
}

\author{
Address: Laboratory of Bacteriology, The Rockefeller University, 1230 York Avenue, New York, NY 10065, USA \\ *Corresponding authors: David Bikard (dbikard@rockefeller.edu) and Luciano A. Marraffini (marraffini@rockefeller.edu) \\ Fl000Prime Reports 2013, 5:47 (doi:10.12703/P5-47) \\ This is an open-access article distributed under the terms of the Creative Commons Attribution-Non Commercial License \\ (http://creativecommons.org/licenses/by-nc/3.0/legalcode), which permits unrestricted use, distribution, and reproduction in any medium, \\ provided the original work is properly cited. You may not use this work for commercial purposes. \\ The electronic version of this article is the complete one and can be found at: http://fl000.com/prime/reports/b/5/47
}

\begin{abstract}
Clustered regularly interspaced short palindromic repeats (CRISPR) loci and their associated cas (CRISPR-associated) genes provide adaptive immunity against viruses (phages) and other mobile genetic elements in bacteria and archaea. While most of the early work has largely been dominated by examples of CRISPR-Cas systems directing the cleavage of phage or plasmid DNA, recent studies have revealed a more complex landscape where CRISPR-Cas loci might be involved in gene regulation. In this review, we summarize the role of these loci in the regulation of gene expression as well as the recent development of synthetic gene regulation using engineered CRISPR-Cas systems.
\end{abstract}

\section{Introduction}

CRISPR-Cas systems have been characterized as a small RNA-based immune system of bacteria and archaea [1-4]. The CRISPR locus harbors an array of short repetitive sequences (30-40 base pairs) separated by equally short "spacer" sequences. CRISPR function was initially inferred from the observation that spacer sequences match the genome of phages and mobile genetic elements of bacteria and archaea [5-7]. Transcription and processing of the repeat-spacer array results in the accumulation of small CRISPR RNAs (crRNAs) that contain a full or partial spacer sequence [8-11]. crRNAs function as a guide that directs a Cas ribonucleoprotein complex to the target sequence (also known as protospacer) [12-15]. Cleavage of phage or plasmid DNA by crRNA:Cas complexes protects cells against viral infection or other invading DNA elements [16-18]. DNA cleavage can be achieved even in the presence of mismatches between the crRNA spacer sequence and the target sequence, a feature that extends the level of protection of a crRNA, that is, different phages with similar but not identical target sequences can be repelled by the CRISPR-Cas system using the same spacer [19]. However, complementarity in a "seed" region, a sequence that is usually 8 to 12 nucleotides (nts) long, is absolutely required for cleavage, and mismatches between the crRNA and its target in this region prevent CRISPR immunity $[19,20]$. A second requirement for cleavage is the presence of a short nucleotide motif flanking the target (known as the protospacer adjacent motif, or PAM) $[11,12,21]$. The PAM sequence is different in differing CRISPR-Cas systems. As a consequence of these two requirements, phages can evade CRISPR immunity by introducing single-nucleotide mutations in either the seed or PAM sequences $[16,21,22]$. Last but not least, a fundamental feature of CRISPR-Cas systems is their ability to capture new spacer sequences from foreign DNA. Cells can gain immunity against infecting viruses or plasmids by incorporating new spacer sequences that match the viral or plasmid genome [16,23-25]. Thus, the spacer sequences of a CRISPR array represent a memory of the viral infections and encounters of the host bacterium with mobile genetic elements.

CRISPR-Cas systems are very diverse: they differ in the number and nature of the associated cas genes, the repeat sequence, and the number of repeat-spacer units. Based on their cas gene content, CRISPR-Cas systems have been classified in three main types, each containing different subtypes [26] that display different mechanisms for the biogenesis of crRNAs and the targeting of the invasive nucleic acid. In type I CRISPR-Cas systems, the crRNA precursor (a long transcript containing all repeats and spacers of the CRISPR array) is processed into small crRNAs by the Cas 6 endoribonuclease associated with a 
complex composed by other Cas proteins named Cascade (CRISPR-associated complex for antiviral defense) [8]. Loaded with the crRNA guide, the Cascade guides the Cas3 nuclease to cleave the DNA of the invader [12]. In type II CRISPR-Cas systems, processing of the crRNA requires Cas9, the tracrRNA (trans-activating crRNA, a small RNA antisense to the repeat sequence), and RNase III (which cleaves the tracrRNA:repeat doublestranded RNA [dsRNA] to liberate small crRNAs, see Figure 1) [11]. Cas9 cleaves dsDNA at sites specified by the crRNA $[14,15]$. Finally, type III systems, similarly to type I systems, utilize Cas 6 to cleave the precursor into small crRNAs [10,27]. However, different subtypes target different substrates. In subtype III-A, DNA is targeted [17], although the nuclease or proteins that execute CRISPR immunity are unknown. In subtype III-B, in vitro experiments have shown that a complex composed of Cmr (Cas module receptor activity-modifying proteins [RAMP]) proteins cleaves RNA molecules that are complementary to the crRNA guide [28]. This diversity in the machinery and function of CRISPR-Cas systems has led to the hypothesis that they might not all have the same function [28]. The common features shared by all CRISPR-Cas systems are (a) the ability to acquire new spacers and (b) the presence of RNA-guided Cas proteins with DNA- and RNA-binding and cleavage activities.

Figure I. Type II CRISPR-Cas systems

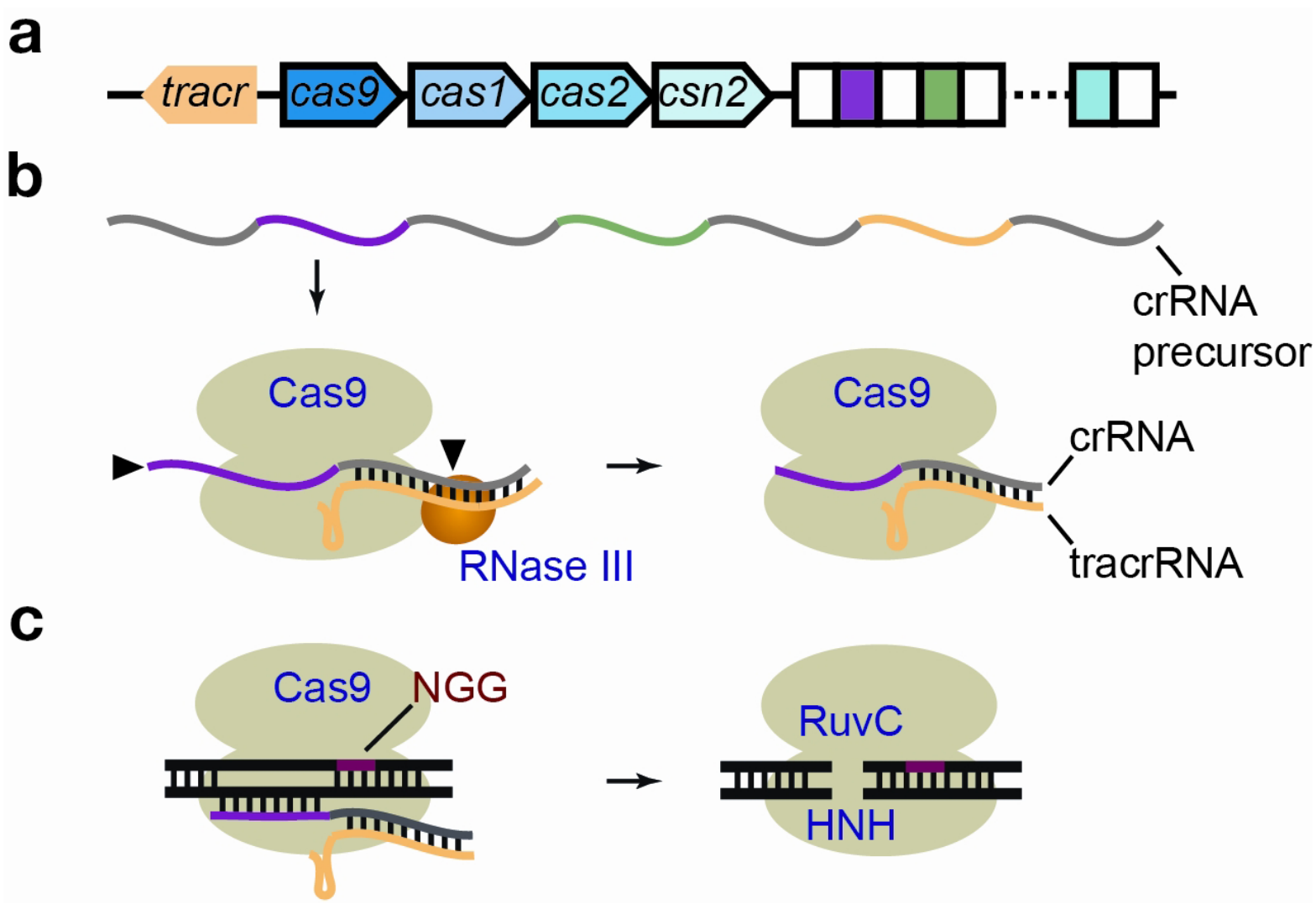

(a) Genetic organization of the type II-A CRISPR-Cas system of Streptococcus pyogenes SF370. The cas operon is composed of four genes, cas9, cas I, cas2, and csn2; the last three are thought to be involved in the acquisition of new spacer sequences. This operon is followed by the CRISPR array containing seven repeats (white boxes, $36 \mathrm{nt}$ long) and six spacers (numbered, colored boxes, $30 \mathrm{nt}$ long) that match different S. pyogenes bacteriophages. Preceding the cas operon and transcribed in the other direction is the trans-activating CRISPR RNA (tracrRNA) gene, which encodes a small RNA with homology to the repeat sequences. (b) CRISPR RNA (crRNA) processing. The CRISPR locus is transcribed as a long precursor (the crRNA precursor) containing repeats (grey line) and spacers (colored lines). Assisted by Cas9, the tracrRNA interacts with each repeat sequence to generate a double-stranded RNA (dsRNA) that is cleaved by RNase III, thus liberating the small crRNAs from the precursor. Further processing at the $5^{\prime}$ end of the crRNA shortens the length of the guide sequence to $20 \mathrm{nt}$. Black arrowheads indicate the sites of RNA processing. (c) Targeting. Cas9 scans the genome ( $d s D N A)$ of the invader to find a region of complementarity with the guide crRNA and introduces a dsDNA cut using two independent nuclease domains, one for each DNA strand, RuvC and HNH. A requirement for cleavage is the presence of an NGG sequence (known as protospacer adjacent motif, or PAM) immediately downstream of the target site. Abbreviations: cas, CRISPR-associated; CRISPR, clustered regularly interspaced short palindromic repeats; $\mathrm{nt}$, nucleotide. 
In principle, these proteins can participate in the regulation of gene expression acting on DNA or RNA molecules. In this review, we will discuss examples of gene regulation by natural CRISPR-Cas systems and then focus on the recent engineering of RNA-guided Cas proteins as tools for the control of gene expression.

\section{Self-targeting spacers}

The existence of self-targeting spacers in CRISPR arrays suggests the possibility that some CRISPR systems might be involved in gene regulation. The presence of such spacers is surprising considering that self-targeting is known to lead to cell death [22,29-31]. A bioinformatics study revealed that approximately $18 \%$ of CRISPRencoding organisms carry a spacer targeting the organism's own genome [32]. However, most of the time, the presence of such spacers can be explained by the mutation or deletion of the cas genes or the absence of a PAM motif that would prevent autoimmunity. Moreover, selftargeting spacers are strongly over-represented among the first spacers of the array, indicating that their acquisition was recent. Together, those facts strongly suggest that the acquisition of a self-targeting spacer is detrimental, leading to autoimmunity, and selects for the loss of CRISPR function.

Experiments in Escherichia coli, where the acquisition machinery alone was overexpressed, have confirmed that the acquisition of self-targeting spacers does occur in vivo [24]. Such events would most likely lead to cell death in the presence of a functional CRISPR-Cas system unless autoimmunity is avoided by the acquisition of spacers whose targets lack a proper PAM. An analysis of the protospacer sequences of the Streptococcus agalactiae CRISPR systems revealed that such PAM-less spacers can be the result of an improper acquisition event in which the spacer is integrated into the CRISPR array in the wrong orientation [33]. It remains to be determined if such spacers could mediate DNA binding without cleavage and affect target transcription and hence gene expression.

The general rule is that functional CRISPR systems do not coexist with a perfect target in the same genome. An exception to this rule might be the case of the type I-E system found in Pelobacter carbinolicus DSM2380 [34]. In this system, the last spacer of a 111 spacer-long array perfectly matches the essential histidyl-tRNA synthetase gene hisS, and the target displays a PAM sequence typical of type I-E systems, AAG [25]. The fact that this hisS targeting spacer is found at the very end of the array suggests that it was acquired a long time ago, while the large size of the array suggests that the system remained active after the hisS-targeting spacer acquisition. This spacer was shown to be transcribed in P. carbinolicus, but the functionality of this CRISPR-Cas system could not be ascertained because of the lack of genetic tools for this bacterium. The authors of this study nevertheless argue that the acquisition of this spacer might have downregulated the expression of the hisS gene, which drove a reduction in the usage of histidines in $P$. carbinolicus proteins compared to closely related organisms.

\section{Gene regulation mediated by small CRISPR-Cas-associated RNA}

The most conclusive example of gene regulation by a CRISPR-Cas system comes from a recent study of the intracellular pathogen Francisella novicida. Gene FTN_0757 was identified in a screen for virulence factors and was shown to repress the production of a bacterial lipoprotein (BLP), which induces an inflammatory response [35]. FTN_0757 turned out to be a member of the Cas9 protein family of the type II CRISPR-Cas systems (Figure 2a) [36]. Whereas, Cas9 and the tracrRNA were required for BLP repression in F. novicida; the CRISPR array was dispensable. The tracrRNA was found to carry approximately an 85-nucleotide region of imperfect complementarity to the BLP messenger that encompasses the start codon (Figure 2b), but is not sufficient to provide repression by itself, as it requires Cas9 as well as a novel CRISPR-associated RNA, the scaRNA (small CRISPRCas-associated RNA) [36,37]. The scaRNA consists of a degenerate CRISPR repeat sequence with an approximate 20-nucleotide region of complementarity to the tracrRNA located immediately upstream of the CRISPR array. Both of these complementarity regions are essential for the Cas9-mediated repression of the BLP gene and, as a consequence of their interactions, the BLP mRNA is degraded. It remains to be determined if Cas9 is directly or indirectly involved in the degradation of mRNA-for example, by recruiting an mRNA nuclease or by blocking translation, which could in turn lead to early termination and mRNA degradation.

Whether crRNAs generated by the CRISPR array in F. novicida can work with this special tracrRNA to direct either interference or regulation remains to be tested. The most likely explanation for the evolution of BLP regulation is that a CRISPR locus involved in the protection against phages and mobile genetic elements was co-opted to perform a new function. Interestingly, Stern and colleagues predicted a similar scenario when considering the possibility of CRISPR-mediated regulation of gene expression [32]. The authors analyzed the possible function of self-targeting spacers-that is, spacers whose sequence matches a sequence present elsewhere in the chromosome-and argued that these were unlikely to be involved in gene regulation because the spacer content of CRISPR loci is too dynamic. Instead, they hypothesized 
Figure 2. CRISPR-mediated regulation of gene expression in Francisella novicida

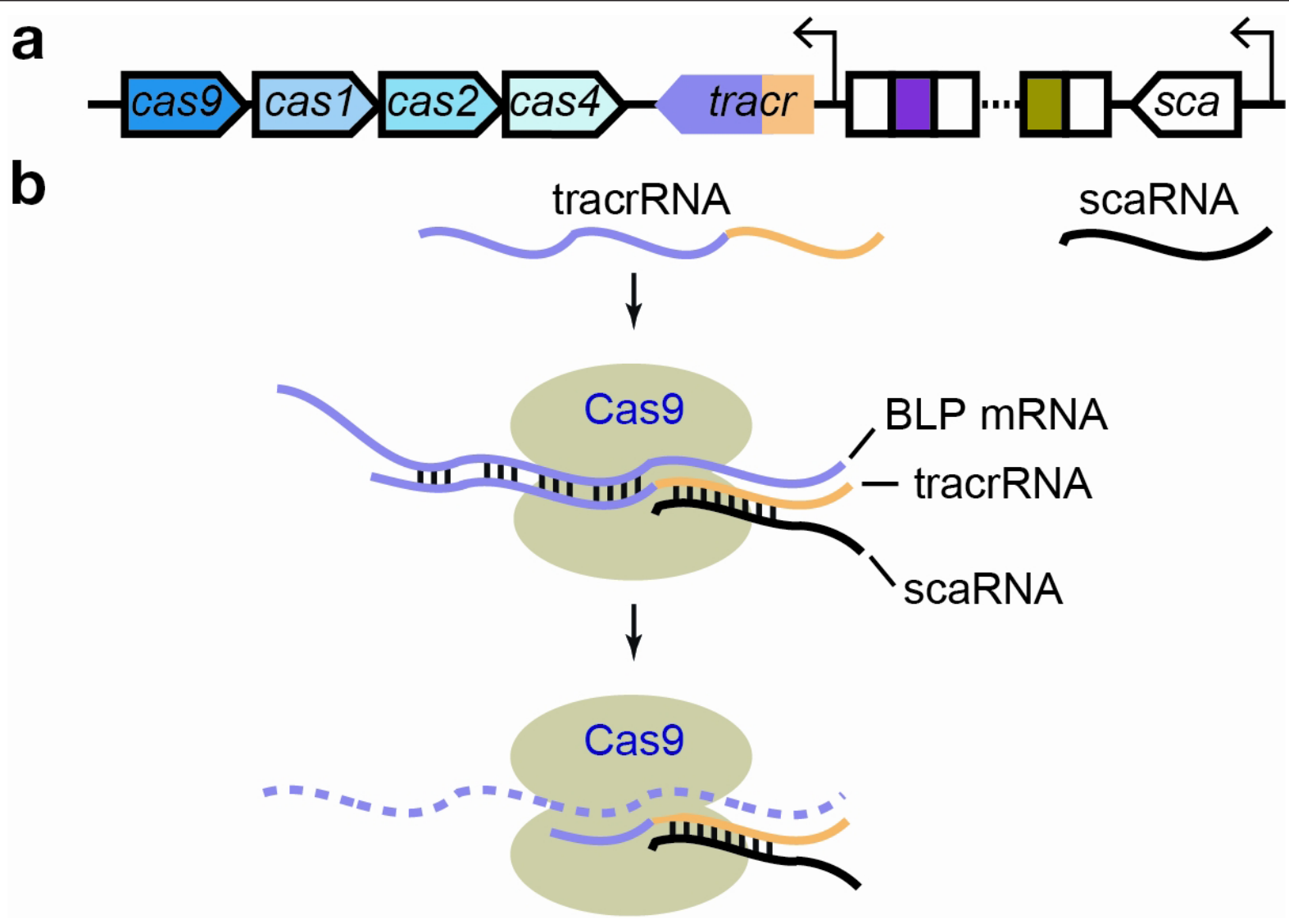

(a) Genetic organization of the type II-B CRISPR-Cas system present in F. novicida. It contains I4 different spacer sequences and cas 4 instead of csn2. Unique to this system is the presence of a small CRISPR-Cas-associated RNA (scaRNA) transcribed in the same orientation as the CRISPR array. (b) Trans-activating CRISPR RNA (tracrRNA):scaRNA-mediated gene repression. The $5^{\prime}$ end of the tracrRNA is homologous to the repeat sequences (yellow line). This allows its interaction with the scaRNA and its incorporation within Cas9. The $3^{\prime}$ end of the tracrRNA has homology to the bacterial lipoprotein (BLP) messenger RNA and directs the degradation/cleavage of this transcript by an unknown mechanism. Abbreviations: cas, CRISPR-associated; CRISPR, clustered regularly interspaced short palindromic repeats.

that CRISPR-mediated gene regulation would have to be encoded in a more stable way if it is to be sustained, and proposed that regulation could be achieved by "atypical" CRISPR structures, possibly composed of a single spacer and altered repeats, making them more stable. This is in essence a description of the tracrRNA found in F. novicida.

An indication that this regulation was selected and is not purely accidental is the fact that the repression of BLP by Cas9 appears to be itself regulated. A 100-fold induction of cas9, tracrRNA, and scaRNA RNAs was observed when bacteria were inside phagosomes, where BLP downregulation allows evasion of the host immune defenses and promotes bacteria survival [36]. While the predominance of tracrRNA:scaRNA-mediated regulation remains to be investigated, its discovery in F. novicida suggests that CRISPR-Cas loci can be easily converted to regulatory elements. A deletion of Cas9 in Neisseria meningitidis affected virulence traits, such as adherence and invasion of human epithelial cells [36], suggesting a broad role for Cas9 in the regulation of pathogenesis.

\section{CRISPR interaction with an imperfect target could mediate gene regulation}

Several studies have suggested that CRISPR interaction with an imperfect target could mediate gene regulation. Early evidence comes from work in the bacterium Pseudomonas aeruginosa, where a type I-F CRISPR-Cas system is involved in the inhibition of biofilm formation [38]. P. aeruginosa PA14 carries two CRISPR systems (CR1 and CR2). The first spacer of CR2 (CR2_sp1) has imperfect complementarity with a gene of the lysogenic phage DMS3 (DMS3-42). The interaction between CR2-sp1 and DMS3-42 was found to be responsible for biofilm 
inhibition in this strain and requires the presence of cas genes in addition to CR2-sp1 [39]. Restoring a perfect complementarity between the spacer and its target resulted in efficient immunity against DMS3 phage infection [23]. It is thus hypothesized that the presence of mismatches between CR2-sp1 and its DMS3-42 target allows for their coexistence in the same cell by preventing effective CRISPR immunity against the lysogen. Curiously, while deletion of DMS3-42 restored biofilm formation in a CRISPRpositive strain, it was also shown that the DMS3-42 protein itself was not required for the inhibition phenotype. How exactly this CRISPR interaction with the DMS3 lysogen inhibits biofilm formation remains a mystery. We believe that inefficient interference due to the mismatches could still lead to some degree of DNA cleavage, inducing repair pathways that might have pleiotropic effects. Alternatively, the expression of genes in the $17 \mathrm{~kb}$ operon, where DMS3-42 is located, could be affected by Casprotein binding either to DNA or to the mRNA and might play a role in biofilm formation.

Other studies have reported phenotypic effects caused by CRISPR interaction with an imperfect target. During a study of the Tat protein secretion system in E. coli, Perez-Rodriguez and colleagues constructed a plasmid carrying a fusion between a Tat signal found in the ssTorA leader peptide and a green fluorescent protein (GFP) reporter [40]. They found that an interaction between the sstorA sequence and a spacer (spacer5) of the E. coli type I-E CRISPR-Cas was responsible for GFP silencing. The observed reduction in fluorescence was actually due to a reduction in the plasmid copy number that was dependent on the cas genes as well as on the homology between spacer 5 and its target. In principle, DNA binding without cleavage of the spacer5 ribonucleoprotein complex to the reporter plasmid could affect its replication or stability. However, the region of homology between spacer 5 crRNA and its target presents none of the characteristics that have been shown to be essential for the binding of the E. coli crRNA-Cas nucleoprotein complex to its target DNA: there is neither homology within the seed sequence [19] nor a proper PAM sequence [12] flanking the sstorA target. Thus, we believe that another hypothesis could be explored: an interaction between the spacer 5 crRNA and the sstorA-gfp reporter mRNA might affect the plasmid copy number.

A final example of a mysterious interaction involving crRNAs occurs in the bacterial pathogen Listeria monocytogenes. Here, an orphan CRISPR array (lacking nearby cas genes) was shown to be expressed but not processed into small crRNAs. This non-coding RNA, named RliB [41], was shown to bind and affect the expression of the Imo2104 and lmo2105 genes, encoding the ferrous iron transport proteins FeoA and $\mathrm{FeoB}$, respectively, and affecting virulence in a mouse model of listeriosis.

\section{RNA-targeting CRISPR systems}

Early predictions of CRISPR-Cas function suggested the possibility that these loci could be involved in gene regulation in a mechanism similar to eukaryotic RNA interference (RNAi) [42]. However, as opposed to RNAi, the target of most CRISPR types was found to be DNA $[17,18]$. A notable exception is the type III-B CRISPR-Cas system of the euryarchaeon Pyrococcus furiosus. In these organisms, a Cas protein complex, also known as CMR complex, was purified [28]. The complex co-purified with processed and matured crRNAs that could direct the cleavage of a target RNA sequence that matches the crRNA guide. A PAM sequence is not necessary for this cleavage [43]. Another type III-B CMR complex purified from Sulfolobus solfataricus was also shown to cleave RNA in vitro [44], adding generality to the notion of RNA targeting CRISPR-Cas systems. Moreover, type III-B CRISPR-Cas systems are frequently found together with other systems in the same genome, suggesting that they may have a distinct function not carried by the other coexisting CRISPR loci. However, it is still unclear whether type III-B CRISPR-Cas systems are involved in viral defense and/or gene regulation as none of the 200 spacers found in the seven CRISPR loci carried by $P$. furiosus have matches in the databases. Because neither known phages nor endogenous genes are targeted, it is difficult to infer their function. An additional conundrum is the acquisition of new spacers by these systems. Unless dsRNA phages are the target, if single-stranded RNA (ssRNA) phages or transcripts are targeted, a mechanism would have to ensure that newly acquired spacers are integrated in the correct orientation to produce a crRNA antisense to the ssRNA genome.

\section{Synthetic control of gene expression by Cas proteins}

Recently, two groups showed that the type II-A CRISPR system of Streptococcus pyogenes, known to direct DNA cleavage, could be engineered to repress gene expression $[45,46]$. The crRNA-guided dsDNA nuclease of this system, Cas9, carries two catalytic sites, a RuvC-like domain and an HNH nuclease domain, each cleaving one strand of the target DNA $[14,15]$. To generate an RNA-guided DNA-binding protein (dCas9), mutations that abolish DNA cleavage were introduced in each domain. dCas9 can be directed to bind its target either by a crRNA in the presence of the tracrRNA or by an engineered single-guide RNA (sgRNA) that consists of a fusion between the tracrRNA and the crRNA [14]. The binding of dCas9 to the promoter region of a reporter gene blocks transcription initiation (Figure 3a), while 
Figure 3. RNA-guided gene repression using engineered CRISPR-Cas systems

a

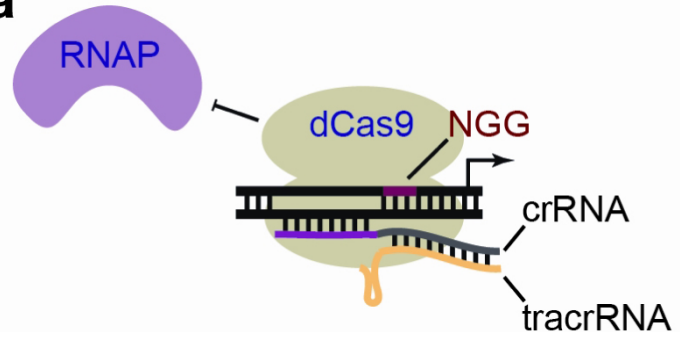

b

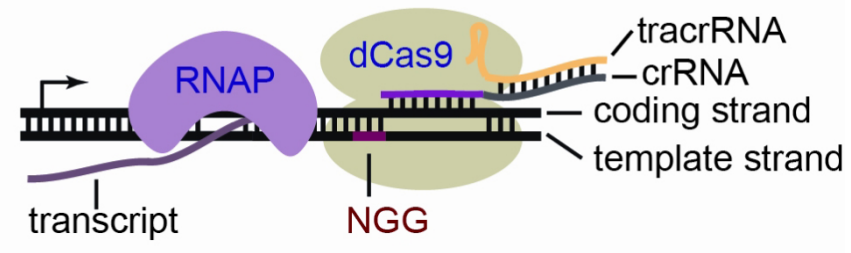

(a) Mutation of the essential residues of the RuvC and HNH nuclease domains converts Cas9 into a CRISPR RNA (crRNA)-guided DNA-binding protein (dCas9). Directing dCas9 to promoter sequences (either the top or bottom DNA strand) results in prevention of transcription initiation, most likely by preventing the binding of RNA polymerase (RNAP) to the promoter elements. (b) Directing dCas 9 to the open reading frame regions of genes (with a crRNA that interacts with the coding but not the template strand) results in a transcription stop, presumably because the dCas 9 complex blocks RNAP elongation. Arrows indicate the transcription start site. Abbreviations: cas, CRISPR-associated; CRISPR, clustered regularly interspaced short palindromic repeats; tracrRNA, trans-activating CRISPR RNA.

binding to the coding strand of the open reading frame was shown to block transcription elongation (Figure $3 \mathrm{~b}$ ) $[45,46]$. Therefore, co-expression of dCas9 and its guide RNA provides a simple way to shut down expression of any gene (specified by the guide RNA sequence) in the genome. While dCas9 can dramatically repress the expression of bacterial genes by itself, the fusion of a chromatin modifier domain (KRAB, Mxi1) to dCas9 was required for efficient repression in eukaryotic cells [47]. When Cas9 is under the control of an inducible promoter, repression can be reversible [46]. In addition, by using multiple guide RNAs, dCas9 can repress several genes at the same time independently $[46,47]$. Finally, RNA-sequencing studies showed the high specificity of this technology, since only the abundance of the transcript of the targeted gene was reduced upon dCas9 expression $[46,47]$. Interestingly, the catalytically active Cas9 can also direct repression in bacteria [45]. While a small number of mismatches between the $5^{\prime}$ end of the crRNA:target interaction were sufficient to abolish the cleavage activity of Cas9, the binding and repression activity were indistinguishable from that of dCas9 in the same conditions. Such repression by wildtype Cas9 could have a role in CRISPR immunity. Target mutations are frequently found in phages that escape CRISPRmediated cleavage. We suggest that the ability of Cas9 to repress gene expression could represent a second line of defense to prevent phage infection by phage "escape" mutants; that is, phages carrying mutations in the target site that prevent Cas9 cleavage will not be able to mount a successful infective cycle if an essential gene is repressed. Furthermore, since there is substantial repression with as little as 10 matches between the crRNA and its target, it is possible that newly acquired spacers can by chance cause repression of host genes. Whether such regulation could, in some instances, be beneficial and selected for is not known.

Finally, dCas9 can also be used to activate transcription, both in mammalian and bacterial cells. In eukaryotes, this was achieved simply by fusing different transcription activator domains (VP64, p65) to dCas9 [44]. In bacteria, dCas9 was fused to the $\omega$ subunit of the RNA polymerase (RNAP) [45], a strategy that was previously used to activate transcription using the DNA-binding activity of the bacteriophage lambda repressor protein $\mathrm{cI}$ [48]. In both cases, the targeting of the dCas9-activator fusion protein to promoter sequences can recruit the RNAP and induce gene expression. Combined with dCas9-mediated repression, this technology offers the possibility to easily control the expression of any gene or combination of genes and will certainly impact on the study of genetic networks and the construction of synthetic systems for biotechnological purposes.

\section{Conclusions}

A large body of evidence has now been accumulated that demonstrates a function for CRISPR-Cas systems as an immune pathway to prevent the infection of phages and other mobile genetic elements. The fact that the large majority of spacers found in CRISPR arrays target foreign sequences suggests that CRISPR-Cas evolution is driven by their function as an immune system rather than by their potential to regulate gene expression. Recent studies, however, clearly established the ability of CRISPR-Cas systems to regulate gene expression by different mechanisms, both in natural and synthetic systems. How profound the impact of this regulation on bacterial physiology is at the moment currently remains unknown. Whether the acquisition of new spacers can be used to regulate the 
expression of bacterial genes is also unknown. If this were the case, CRISPR-Cas systems would offer a simple mechanism for the adaptation to changing environments through the creation of novel regulations. Finally, it is important to note that only a very small fraction of the CRISPR-Cas systems have been carefully studied. The great diversity of CRISPR loci is likely to offer many surprises.

\section{Abbreviations}

BLP, bacterial lipoprotein; Cas, CRISPR-associated; Cmr, Cas module RAMP; CRISPR, clustered regularly interspaced short palindromic repeats; crRNA, CRISPR RNA; dsDNA, double-stranded DNA; nt, nucleotide; PAM, protospacer adjacent motif; RAMP, repeat-associated mysterious proteins; RNAi, RNA interference; RNAP, RNA polymerase; scaRNA, small CRISPR-associated RNA; ssRNA, single-stranded RNA; tracrRNA, transactivating CRISPR RNA.

\section{Disclosures}

The authors declare that they have no competing interests.

\section{Acknowledgments}

David Bikard is supported by a Harvey L. Karp Discovery Award and the Bettencourt Schuller Foundation. LAM is supported by the Searle Scholars Program, the Rita Allen Scholars Program, an Irma T. Hirschl Award, a Sinsheimer Foundation Award, and a National Institutes of Health Director's New Innovator Award (1DP2AI104556-01).

\section{References}

I. Horvath P, Barrangou R: CRISPR/Cas, the immune system of bacteria and archaea. Science 2010, 327:167-70.

2. Wiedenheft B, Sternberg SH, Doudna JA: RNA-guided genetic silencing systems in bacteria and archaea. Nature 2012, 482:331-8.

3. Terns MP, Terns RM: CRISPR-based adaptive immune systems. Curr Opin Microbiol 20II, I4:321-7.

4. Deveau H, Garneau JE, Moineau S: CRISPR/Cas system and its role in phage-bacteria interactions. Annu Rev Microbiol 2010, 64:475-93.

5. Bolotin A, Quinquis B, Sorokin A, Ehrlich SD: Clustered regularly interspaced short palindrome repeats (CRISPRs) have spacers of extrachromosomal origin. Microbiology (Reading, Engl.) 2005, | 5 |:255|-6|

\section{FlOOOPrime}

\section{RECOMMENDED}

6. Mojica FJM, Díez-Villaseñor C, García-Martínez J, Soria E: Intervening sequences of regularly spaced prokaryotic repeats derive from foreign genetic elements. J Mol Evol 2005, 60:174-82.

\section{FlOOOPrime}

\section{RECOMMENDED}

7. Pourcel C, Salvignol G, Vergnaud G: CRISPR elements in Yersinia pestis acquire new repeats by preferential uptake of bacteriophage DNA, and provide additional tools for evolutionary studies. Microbiology (Reading, Engl.) 2005, I 5 I:653-63.

8. Brouns SJJ, Jore MM, Lundgren M, Westra ER, Slijkhuis RJH, Snijders APL, Dickman MJ, Makarova KS, Koonin EV, van der
Oost J: Small CRISPR RNAs guide antiviral defense in prokaryotes. Science 2008, 32 1:960-4.

\section{FIOOOPrime}

9. Haurwitz RE, Jinek M, Wiedenheft B, Zhou K, Doudna JA: Sequenceand structure-specific RNA processing by a CRISPR endonuclease. Science 2010, 329:1355-8.

\section{FlOOOPrime}

RECOMMENDED

10. Hatoum-Aslan A, Maniv I, Marraffini LA: Mature clustered, regularly interspaced, short palindromic repeats RNA (crRNA) length is measured by a ruler mechanism anchored at the precursor processing site. Proc Natl Acad Sci USA 2011, 108:21218-22.

II. Deltcheva E, Chylinski K, Sharma CM, Gonzales K, Chao Y, Pirzada ZA, Eckert MR, Vogel J, Charpentier E: CRISPR RNA maturation by trans-encoded small RNA and host factor RNase III. Nature 20I I, 47I:602-7.

\section{FlOOOPrime \\ RECOMMENDED}

12. Westra ER, van Erp PBG, Künne T, Wong SP, Staals RHJ, Seegers CLC, Bollen S, Jore MM, Semenova E, Severinov K, de Vos WM, Dame RT, de Vries R, Brouns SJJ, van der Oost J: CRISPR immunity relies on the consecutive binding and degradation of negatively supercoiled invader DNA by Cascade and Cas3. Mol Cell 2012, 46:595-605.

13. Wiedenheft B, Lander GC, Zhou K, Jore MM, Brouns SJJ, van der Oost J, Doudna JA, Nogales E: Structures of the RNA-guided surveillance complex from a bacterial immune system. Nature 20II, 477:486-9.

\section{FlOOOPrime}

RECOMMENDED

14. Jinek M, Chylinski K, Fonfara I, Hauer M, Doudna JA, Charpentier E: A programmable dual-RNA-guided DNA endonuclease in adaptive bacterial immunity. Science 2012, 337:816-2I.

\section{FlOOOPrime} RECOMMENDED

15. Gasiunas G, Barrangou R, Horvath P, Siksnys V: Cas9-crRNA ribonucleoprotein complex mediates specific DNA cleavage for adaptive immunity in bacteria. Proc Natl Acad Sci USA 2012, 109:E2579-86

\section{FlOOOPrime}

\section{RECOMMENDED}

16. Barrangou R, Fremaux C, Deveau H, Richards M, Boyaval P, Moineau S, Romero DA, Horvath P: CRISPR provides acquired resistance against viruses in prokaryotes. Science 2007, 3 I 5: I 709- 12.

\section{FlOOOPrime
RECOMMENDED}

17. Marraffini LA, Sontheimer EJ: CRISPR interference limits horizontal gene transfer in staphylococci by targeting DNA. Science 2008, 322:1843-5.

\section{FlOOOPrime
RECOMMENDED}

18. Garneau JE, Dupuis M, Villion M, Romero DA, Barrangou R, Boyaval P, Fremaux C, Horvath P, Magadán AH, Moineau S: The CRISPR/Cas bacterial immune system cleaves bacteriophage and plasmid DNA. Nature 2010, 468:67-7I.

\section{FlOOOPrime}

\section{RECOMMENDED}

19. Semenova E, Jore MM, Datsenko KA, Semenova A, Westra ER, Wanner B, van der Oost J, Brouns SJJ, Severinov K: Interference by clustered regularly interspaced short palindromic repeat (CRISPR) RNA is governed by a seed sequence. Proc Natl Acad Sci USA 201 I, 108:10098-103.

20. Wiedenheft B, van Duijn E, Bultema JB, Bultema J, Waghmare SP, Waghmare S, Zhou K, Barendregt A, Westphal W, Heck AJR, Heck A, 
Boekema EJ, Boekema E, Dickman MJ, Dickman M, Doudna JA: RNAguided complex from a bacterial immune system enhances target recognition through seed sequence interactions. Proc Natl Acad Sci USA 20I I, 108:10092-7.

21. Deveau H, Barrangou R, Garneau JE, Labonté J, Fremaux C, Boyaval P, Romero DA, Horvath P, Moineau S: Phage response to CRISPRencoded resistance in Streptococcus thermophilus. J Bacteriol 2008, 190:1390-400.

\section{FlOOOPrime}

\section{RECOMMENDED}

22. Bikard D, Hatoum-Aslan A, Mucida D, Marraffini LA: CRISPR interference can prevent natural transformation and virulence acquisition during in vivo bacterial infection. Cell Host Microbe 2012, I 2:177-86

\section{FlOOOPrime RECOMMENDED}

23. Cady KC, Bondy-Denomy J, Heussler GE, Davidson AR, O'Toole GA: The CRISPR/Cas adaptive immune system of Pseudomonas aeruginosa mediates resistance to naturally occurring and engineered phages. J Bacteriol 2012, 194:5728-38.

24. Yosef I, Goren MG, Qimron U: Proteins and DNA elements essential for the CRISPR adaptation process in Escherichia coli. Nucleic Acids Res 2012, 40:5569-76.

\section{FlOOOPrime}

25. Datsenko KA, Pougach K, Tikhonov A, Wanner BL, Severinov K, Semenova E: Molecular memory of prior infections activates the CRISPR/Cas adaptive bacterial immunity system. Nat Commun 2012, 3:945.

\section{FlOOOPrime} RECOMMENDED

26. Makarova KS, Haft DH, Barrangou R, Brouns SJ], Charpentier E, Horvath P, Moineau S, Mojica FJM, Wolf YI, Yakunin AF, van der Oost J, Koonin EV: Evolution and classification of the CRISPRCas systems. Nat Rev Microbiol 20I I, 9:467-77.

\section{FlOOOPrime \\ RECOMMENDED}

27. Carte J, Wang R, Li H, Terns RM, Terns MP: Cas6 is an endoribonuclease that generates guide RNAs for invader defense in prokaryotes. Genes Dev 2008, 22:3489-96.

28. Hale CR, Zhao P, Olson S, Duff MO, Graveley BR, Wells L, Terns RM, Terns MP: RNA-guided RNA cleavage by a CRISPR RNA-Cas protein complex. Cell 2009, 139:945-56.

\section{FlOOOPrime}

29. Jiang W, Bikard D, Cox D, Zhang F, Marraffini LA: RNA-guided editing of bacterial genomes using CRISPR-Cas systems. Nat Biotechnol 2013, 31:233-9.

30. Edgar R, Qimron U: The Escherichia coli CRISPR system protects from $\lambda$ lysogenization, lysogens, and prophage induction. J Bacteriol 2010, 192:629I-4.

31. Paez-Espino D, Morovic W, Sun CL, Thomas BC, Ueda K, Stahl B, Barrangou R, Banfield JF: Strong bias in the bacterial CRISPR elements that confer immunity to phage. Nat Commun 20I3, $4: 1430$

32. Stern A, Keren L, Wurtzel O, Amitai G, Sorek R: Self-targeting by CRISPR: gene regulation or autoimmunity? Trends Genet 2010, 26:335-40.

\section{FlOOOPrime}

\section{RECOMMENDED}

33. Lopez-Sanchez M, Sauvage E, Da Cunha V, Clermont D, Ratsima Hariniaina E, Gonzalez-Zorn B, Poyart C, Rosinski-Chupin I, Glaser P: The highly dynamic CRISPRI system of Streptococcus agalactiae controls the diversity of its mobilome. Mol Microbiol 20I2, 85: |057-7I.
34. Aklujkar M, Lovley DR: Interference with histidyl-tRNA synthetase by a CRISPR spacer sequence as a factor in the evolution of Pelobacter carbinolicus. BMC Evol Biol 2010, 10:230.

35. Jones CL, Sampson TR, Nakaya HI, Pulendran B, Weiss DS: Repression of bacterial lipoprotein production by Francisella novicida facilitates evasion of innate immune recognition. Cell Microbiol 2012, 14:1531-43.

36. Sampson TR, Saroj SD, Llewellyn AC, Tzeng Y, Weiss DS: A CRISPR/ Cas system mediates bacterial innate immune evasion and virulence. Nature 20I3, 497:254-7.

\section{FIOOOPrime}

37. Sampson TR, Saroj SD, Llewellyn AC, Tzeng Y, Weiss DS: Corrigendum: A CRISPR/Cas system mediates bacterial innate immune evasion and virulence. Nature 2013.

38. Zegans ME, Wagner JC, Cady KC, Murphy DM, Hammond JH, O'Toole GA: Interaction between bacteriophage DMS3 and host CRISPR region inhibits group behaviors of Pseudomonas aeruginosa. J Bacteriol 2009, I 9 I:21 0-9.

39. Cady KC, O'Toole GA: Non-identity-mediated CRISPRbacteriophage interaction mediated via the Csy and Cas3 proteins. J Bacteriol 20II, 193:3433-45.

\section{FlOOOPrime} RECOMMENDED

40. Perez-Rodriguez R, Haitjema C, Huang Q, Nam KH, Bernardis S, Ke A DeLisa MP: Envelope stress is a trigger of CRISPR RNA-mediated DNA silencing in Escherichia coli. Mol Microbiol 201 I, 79:584-99.

4I. Mandin P, Repoila F, Vergassola M, Geissmann T, Cossart P: Identification of new noncoding RNAs in Listeria monocytogenes and prediction of mRNA targets. Nucleic Acids Res 2007, 35:962-74.

\section{FlOOOPrime
RECOMMENDED}

42. Makarova KS, Grishin NV, Shabalina SA, Wolf YI, Koonin EV: A putative RNA-interference-based immune system in prokaryotes: computational analysis of the predicted enzymatic machinery, functional analogies with eukaryotic RNAi, and hypothetical mechanisms of action. Biol Direct 2006, I:7.

\section{FlOOOPrime
RECOMMENDED}

43. Hale CR, Majumdar S, Elmore J, Pfister N, Compton M, Olson S, Resch AM, Glover CVC, Graveley BR, Terns RM, Terns MP: Essential features and rational design of CRISPR RNAs that function with the Cas RAMP module complex to cleave RNAs. Mol Cell 2012, 45:292-302.

44. Zhang J, Rouillon C, Kerou M, Reeks J, Brugger K, Graham S, Reimann J, Cannone G, Liu H, Albers S, Naismith JH, Spagnolo L, White MF: Structure and mechanism of the CMR complex for CRISPRmediated antiviral immunity. Mol Cell 2012, 45:303-13.

45. Bikard D, Jiang W, Samai P, Hochschild A, Zhang F, Marraffini LA Programmable repression and activation of bacterial gene expression using an engineered CRISPR-Cas system. Nucleic Acids Res 2013, 41:7429-37.

46. Qi LS, Larson MH, Gilbert LA, Doudna JA, Weissman JS, Arkin AP, Lim WA: Repurposing CRISPR as an RNA-guided platform for sequence-specific control of gene expression. Cell 2013, 152:1173-83.

\section{FlOOOPrime}

\section{RECOMMENDED}

47. Gilbert LA, Larson MH, Morsut L, Liu Z, Brar GA, Torres SE, Stern-Ginossar N, Brandman O, Whitehead EH, Doudna JA, Lim WA, Weissman JS, Qi LS: CRISPR-mediated modular RNA-guided regulation of transcription in eukaryotes. Cell 20I3, I54:442-5I.

\section{FlOOOPrime
RECOMMENDED}

48. Dove SL, Hochschild A: Conversion of the omega subunit of Escherichia coli RNA polymerase into a transcriptional activator or an activation target. Genes Dev 1998, 12:745-54. 